\title{
Kernos
}

Revue internationale et pluridisciplinaire de religion grecque antique

30 | 2017

Varia

\section{Hellenistic Sanctuaries Between Greece and Rome}

\section{Alaya Palamidis}

\section{OpenEdition \\ Journals}

\section{Édition électronique}

URL : http://journals.openedition.org/kernos/2521

DOI : 10.4000/kernos.2521

ISSN : 2034-7871

\section{Éditeur}

Centre international d'étude de la religion grecque antique

\section{Édition imprimée}

Date de publication : 1 octobre 2017

Pagination : 332-334

ISSN : 0776-3824

\section{Référence électronique}

Alaya Palamidis, « Hellenistic Sanctuaries Between Greece and Rome », Kernos [En ligne], 30 | 2017, mis en ligne le 01 octobre 2017, consulté le 24 septembre 2020. URL : http://journals.openedition.org/ kernos/2521; DOI : https://doi.org/10.4000/kernos.2521

Ce document a été généré automatiquement le 24 septembre 2020.

Kernos 


\title{
Hellenistic Sanctuaries Between Greece and Rome
}

\author{
Alaya Palamidis
}

\section{RÉFÉRENCE}

Milena MELFI, Olympia вовоu (éd.), Hellenistic Sanctuaries Between Greece and Rome, Oxford, Oxford University Press, 2016. 1 vol. 13,5 × 21,6 cm, XVI+236 p. ISBN : 978-0-19-9654130 .

1 Cet ouvrage, issu d'un colloque organisé à Oxford les 22 et 23 septembre 2010, a pour ambition de tenter de combler le relatif désintérêt de la recherche pour l'évolution des anciens sanctuaires à l'époque hellénistique. Dans le sillage des études récentes remettant en question l'idée d'un déclin de la religion civique à partir de cette époque, il s'agit d'identifier les différents acteurs à l'œuvre dans les sanctuaires, qui peuvent expliquer les spécificités de la période comprise entre 300 av. J.-C. et 100 ap. J.-C., et de vérifier dans quelle mesure il est possible de parler de continuité et de changement par rapport aux époques précédentes.

2 À contre-pied des études insistant sur l'inventivité de la sculpture hellénistique, Joannis Mylonopoulos démontre ainsi que, dans le cas de l'iconographie mais également de la mise en scène des statues divines, les innovations ont souvent leurs racines à l'époque classique. Le langage visuel des $\mathrm{V}^{\mathrm{e}}$ et $\mathrm{IV}^{\mathrm{e}}$ siècles, perfectionné au fil du temps, est ainsi repris et adapté sans qu'une rupture avec les traditions antérieures ne soit nécessaire. Par ailleurs, les évolutions de la statuaire hellénistique ne permettent pas, selon l'A., d'attester des changements d'ordre religieux. Quant aux nouvelles pratiques dédicatoires, étudiées par Olympia Bobou et qui peuvent elles aussi trouver des antécédents à l' époque classique, elles suggèrent dans certains cas des modificationsdans le culte d'une divinité, comme dans le cas d'Artémis, progressivement associée aux enfants de tous âges et plus seulement aux jeunes filles avant leur mariage. Mais la généralisation, à 
partir du IV siècle, de la pratique de dédier des reliefs représentant des divinités, ainsi que les dédicaces de statues d'enfants et d'offrandes sans lien apparent avec la divinité honorée, s'expliqueraient en partie par l'utilisation croissante des sanctuaires comme des galeries d'art, où les œuvres d'artistes côtoient des offrandes privées destinées principalement à exposer la piété et l'influence des dédicante-s.

3 La grande visibilité des acteurs privés au sein des lieux de culte est en effet l'une des caractéristiques de époque hellénistique et se traduit notamment par la multiplication des actes d' évergétisme. La contribution d'Yves Lafond s'intéresse ainsi à la façon dont les inscriptions honorifiques dans les sanctuaires du Péloponnèse peuvent à la fois servir de vitrine aux élites honorées pour leur piété et leur générosité, et profiter aux cités elles-mêmes, qui y voient un moyen d'affirmer leur identité tout en valorisant des comportements conformes aux traditions. Maria Kantirea étudie plus précisément le cas du sanctuaire de Despoina à Lykosoura, dépendant des élites locales pour assurer le financement du culte et l'entretien du site. La construction par un évergète d'un temple dédié au culte impérial - à Lykosoura comme le suppose l'A. ou à Mégalopolis - atteste que, outre les bénéfices pour le sanctuaire ou le généreux donateur, un acte d' évergétisme permetégalement d'établir des relations entre la communauté locale et le pouvoir impérial. L'

étude prosopographique des inscriptions du sanctuaire de Despoina révèle enfin 1 'existence d'un réseau de familles aristocratiques du Péloponnèse qui portent un intérêt particulier au culte. La prédominance des élites peut également être reconnue dans la disposition architecturale des sanctuaires, comme le suggère l' étude de Luigi Caliò. L'A. compare l'aspect du sanctuaire de l'acropole de Camiros, dédié à Athéna et Zeus, à celui du sanctuaire d'Hestia, au cœur de la ville basse, tous deux reconstruits au lendemain du séisme de 228-227 av.J.-C., avec le soutien financier probable des Ptolémées. Dans le premier, le nombre important de salles de banquets s'expliquerait par l'organisation d'événements qui concernent l'ensemble de la communauté. Au contraire, le sanctuaire d'Hestia est caractérisé par une succession d'espaces cloisonnés, qui, avec le nombre restreint de klinai, indiquerait que les banquets qui y ont lieu sont réservés à une élite. L'architecture du sanctuaire refléterait ainsi la mise en place d'une démocratie oligarchique, les principales magistratures devenant héréditaires.

4 La prise en charge du financement de certains lieux de culte par des évergètes n'exclut pas une intervention des cités dans d'autres sanctuaires. Björn Forsén propose ainsi d'attribuer la phase de construction hellénistique du sanctuaire ménalien d'Artémis Lykoatis à Mégalopolis, qui aurait administré le sanctuaire après le synécisme de 368367. L'intérêt de cette cité pour le lieu de culte s'expliquerait notamment par la proximité de l'endroit où auraient à l'origine été trouvés les ossements d'Arkas, fils de Kallisto, personnage fondateur de l'identité arcadienne, mais aussi parce qu'il lui permet d'affirmer sa mainmise sur une partie de la Ménalie. L' étude de Sofia Kravaritou offre un aperçuplus complet des cultes d'une cité fondée par synécisme, celle de Démétrias au début du $\mathrm{III}^{\mathrm{e}}$ siècle. Bien que certains sanctuaires de communautés ayant pris part au synécisme soient abandonnés à cette date, d'autres cultes sont repris dans la nouvelle polis. De nouveaux cultes sont également introduits, parmi lesquels celui des archegetai et ktistai, qui serait destiné conjointement aux anciens héros locaux et aux fondateurs royaux de Démétrias, permettrait de fédérer la 
population hétérogène en créant une identité cultuelle commune dans cette cité multiculturelle où Macédoniens et étrangers côtoient les populations locales, thessaliennes et magnésiennes. Mais l'étude permet surtout de mettre en évidence la multiplicité

des acteurs qui interviennent dans la constitution du paysage religieux de la cité : outre la polis et ses dèmes, on note ainsi l'intervention des fondateurs Démétrios Poliorcète et son fils Antigone, honorés pour leur respect des cultes locaux, puis plus tard celle du koinon des Magnésiens ; le Sérapieion est géré par une association cultuelle composée de Grecs comme d'étrangers, tandis que des cultes domestiques sontégalement connus.

5 Àces acteurs locaux s'ajoutent souvent des acteurs régionaux ou suprarégionaux. Jessica Piccinini s'intéresse ainsi au développement architectural du sanctuaire de Dodone au début de l' époque hellénistique, qui coïncide avec le déclin apparent de la fonction oraculaire du lieu de culte et àsa perte d'importance au niveau panhellénique à la même époque. Ce paradoxe s'expliquerait par la nouvelle fonction politique du sanctuaire, qui devient alors le lieu de réunion et de conservation des archives du koinon des Molosses puis des Épirotes. Les naiskoi du sanctuaire, qui constitueraient des trésors plutôt que des temples, seraient des offrandes de la part de différentes puissances régionales ou extérieures à la région : Pyrrhos, les Molosses, les Chaones, Philippe $\mathrm{V}$, mais aussi Corcyre et peut-être Athènes. À Cos, Elisabetta Interdonato note la grande continuité architecturale et cultuelle qui peut être observée dans l'Asklépieion tout au long des périodes hellénistique et impériale, malgré le fait que le sanctuaire soit successivement placé dans la sphère d'influence des Ptolémées, des Attalides et des Romains, qui sont honorés à cet endroit et qui, pour les deux premiers, financent probablement des travaux de constructions rendant possible l'organisation d'importants jeux pentétériques à dimension panhellénique.

6 Àpartir duII ${ }^{\mathrm{e}}$ siècle, l'influence ou la présence des Romains est perceptible dans de nombreux sanctuaires. Milena Melfi, qui étudie les interventions de Damophon sur la côte et dans les îles ioniennes, suggère ainsi que Bouthrôtos et Leukas font appel au sculpteur autour des années $160 \mathrm{av}$. J.-C., après avoir acquis une importance politique nouvelle sous l'égide de Rome.ÀLeukas etàCéphallénie, Damophon aurait restauré ou créédes statues d'Aphrodite Aineias et de Zeus Aine(s)ios respectivement. Le choix de promouvoir ces cultes à cette époque s'expliquerait par les épiclèses divines, qui, en étant mises en lien avec Énée, auraient permis de renforcer les relations de ces îles avec Rome. Damophon, par son néoclassicisme, aurait été idéalement placé pour concilier le besoin de ces cités d'affirmer leur identité tout en reconnaissant la nécessité d'accepter la transition vers une nouvelle époque. Annalisa Lo Monaco s'intéresse elle aussi à la façon dont les sanctuaires réagissent à la présence romaine, en redédicaçant notamment des œuvres anciennes mais aussi des fêtes à leurs nouveaux bienfaiteurs. Mais les Romains sont eux-mêmes acteurs dans les sanctuaires, comme le montrent les offrandes de couronnes, traditionnellement offertes comme cadeaux diplomatiques, ou de boucliers, qui permettent aux généraux romains de se féliciter d'une victoire militaire en remerciant les divinités adjuvantes. À partir de la conquête de Lucius Mummius, la présence des Romains devient plus manifeste puisqu'ils construisent des monuments, redédicacent des statues voire déplacent des offrandes d'un sanctuaire à un autre. 
7 Les deux dernières contributions s'attachent au sort des anciens sanctuaires grecs situés à l'emplacement d'une colonie romaine. À Corinthe, un siècle après la destruction de la ville, les sanctuaires urbains ne sont pas reconstruits après la fondation de la colonie en 44 av. J.-C. Milena Melfi s'intéresse plutôt aux sanctuaires extra-urbains d'Asklépios, de Déméter et Koré et d'Aphrodite, qui sont à nouveau fréquentés, probablement parce qu'il s'agit de cultes qui répondent aux besoins des nouveaux habitants et qui peuvent s'adresser à la fois aux Romains et aux Grecs. Le nombre important d'esclaves grecs affranchis parmi la population expliquerait une continuité dans les pratiques cultuelles à l'origine, rapidement suivie d'une romanisation des cultes à partir de l'époque augustéenne. Dans le sanctuaire de Déméter et Koré, l'élément magique, absent précédemment, serait à mettre en lien avec le rituel de la devotio, par lequel les Romains se seraient approprié le territoire corinthien conquis. Lorenzo Campagna étudie quant à lui le sanctuaire hellénistique situé près de l'église de Santa Caterina,àTauromenion. La construction, probablementàl 'époque augustéenne, d'un mur qui dissimule la façade du temple en séparant le lieu de culte de l'agora, serait liée à une volonté de déprécier les anciens sanctuaires en représailles du choix de la cité de soutenir Pompée lors du conflit qui opposa ce dernier à Octavien. Le sanctuaire aurait alors été progressivement abandonné, et l'intégration du temple dans l'édifice théâtral construit auII ${ }^{\mathrm{e}}$ siècle aurait eu une valeur uniquement muséale.

8 L'ouvrage est stimulant mais on peut regretter qu'il s'agisse principalement d'une succession d'études de cas qui, surtout en l'absence d'une conclusion générale, ne sont pas nécessairement représentatifs de l'ensemble des sanctuaires grecs. Ainsi, la question posée dans l'introduction (p.4), à savoir si le concept de «polis-religion »est utile à la compréhension de la religion hellénistique, n'est pas suffisamment traitée puisque la polis est assez peu présente au fil des pages. Le volume n'en demeure pas moins très intéressant pour quiconque s'intéresse à la religion de cette période.

\section{AUTEURS}

ALAYA PALAMIDIS

(Université de Liège) 